\title{
A molecular model for diacylglycerol acyltransferase from Mortierella ramanniana var. angulispora
}

\author{
Sanjay Mishra ${ }^{1, *}$, Surya Prakash Dwivedi ${ }^{1}$, Neeraja Dwivedi ${ }^{1}$, Ajay Kumar ${ }^{1}$, Anil Rawat ${ }^{2}$ and Yasushi Kamisaka ${ }^{3}$ \\ ${ }^{1}$ Department of Biotechnology, College of Engineering and Technology, IFTM Campus, Lodhipur-Rajput, Delhi Road, Moradabad 244 001, \\ U.P., India; ${ }^{2}$ Bioinformatics Laboratory, Biobranz Institute, Lucknow 226001 , U.P., India; ${ }^{3}$ Lipid Engineering Research Group Institute for \\ Biological Resources and Functions, National Institute of Advanced Industrial Science and Technology, Tsukuba, Ibaraki 305-8566 Japan; \\ Sanjay Mishra - Email: sanjaymishra66@gmail.com
}

Received December 10, 2008; revised February 19 2009; accepted April 14, 2009; published June 28, 2009

\begin{abstract}
:
Acyl CoA diacylglycerol acyltransferase (DGAT, EC 2.3.1.20) is recognized as a key player of cellular diacylglycerol metabolism. It catalyzes the terminal, yet the committed step in triacylglycerol synthesis using diacylglycerol and fatty acyl CoA as substrates. The protein sequence of diacylglycerol acyltransferse (DGAT) Type 2B in Moretierella ramanniana var. angulispora (Protein_ID = AAK84180.1) was retrieved from GenBank. However, a structure is not yet available for this sequence. The 3D structure of DGAT Type 2B was modeled using a template structure (PDB ID: 1K30) obtained from Protein databank (PDB) identified by searching with position specific iterative BLAST (PSI-BLAST). The template (PDB ID: 1K30) describes the structure of DGAT from Cucurbita moschata. Modeling was performed using Modeller 9v2 and protein model is hence generated. The DGAT type 2B protein model was subsequently docked with six inhibitors (sphingosine; trifluoroperazine; phosphatidic acid; lysophospatidylserine; $\mathrm{KCl}$; 1, 2-diolein) using AutoDock (a molecular docking program). The binding of inhibitors to the protein model of DGAT type $2 \mathrm{~B}$ is discussed.
\end{abstract}

Key words: AutoDock; DGAT; diacylglycerol (DG); Mortierella ramanniana; Modeller; PDB; phosphatidic acid (PA); PSIBLAST; RMSD; triacylglycerol (TG)

\section{Background:}

The enzyme diacylglycerol acyltransferase (DGAT) [EC 2.3.1.20] catalyses the chain terminating step in triacylglycerol (TG) biosynthesis. This utilizes diacylglycerol (DG) as a substrate in competition with phospholipids biosynthesis through the Kennedy pathway. TG and phospholipids thus synthesized have distinct physicochemical characteristics that could have an impact on intracellular membrane biogenesis. The fatty acid profile of these lipids is well maintained due to the substrate specificities of DGAT and DG-utilizing enzymes through deacylation-reacylation of these lipids. TG is potentially synthesized and sequestered in spatial cells such as hepatocytes, adipocytes, plant seeds and oleaginous fungi [1 4]. DGAT is assumed to be involved in the specific augmentation of TG accumulation in these types of cells. Manipulation of DGAT could regulate the quality and quantity of TG. However, the molecular role of DGAT is still unclear despite its important role in TG associated disorder of mammalian cells. A very few reports describe its purification [5 - 7], and the purified fraction contained several peptides, among which the catalytic subunit remains to be identified. Mortierella ramanniana var. angulispora is known to accumulate lipids (especially TG). TG contains relatively large amount of $\gamma$-linolenic acid. The fungus is known as a potent producer of $\gamma$-linolenic acid and essential fatty acids by fermentation [8 -10]. Such oleaginous fungi are expected to exhibit amplified expression of specific mechanisms for TG biosynthesis and TG sequestration in lipid bodies taking advantage of oleaginicity. DGAT has been characterized in

ISSN 0973-2063 (online) 0973-8894 (print) Bioinformation 3(9): 394-398 (2009) this fungus to explain the mechanism of TG biosynthesis and sequestration [11, 12]. DGAT activity was higher in the lipid body fraction as compared to that in membrane fraction. It has also been found that DGAT is activated by specific anionic phospholipids [13] providing insights to the regulation. We reported the purification of DGAT from the lipid body fraction of the fungus [14]. This allowed for further studies on TG biosynthesis. Here, we describe the binding of the inhibitors with the protein model of DGAT type $2 \mathrm{~B}$.

\section{Methodology: \\ DGAT sequence}

The sequence of the diacylglycerol acyltransferse (DGAT) type 2B from Mortierella ramanniana var. angulispora (Protein_ID = AAK84180.1) comprising of 355 residues was retrieved from GenBank.

\section{Template structure selection}

A suitable template (PDB-ID: 1K30) was selected using PSIBLAST [15] search with PDB. The selected template is DGAT from Cucurbita moschata with more than 70\% sequence similarity.

\section{Modeling of DGAT type 2B}

The template (1K30) and target (DGAT Type 2B) sequences were aligned using mGenThreader Server. [16] After careful examination for the potential alignment errors, the automated comparative protein modeling program MODELLER 9v2 [17] was employed to build the model. 


\section{Structure submission to Protein Model Database:}

The molecular configuration of DGAT is investigated using data described elsewhere [14]. The authors thus submitted the two protein models for DGAT as follows: (a) DGAT 2B model submitted to Protein Model Database in France during August 2007 (Protein ID: PM 0074978) and (B) DGAT 2A model submitted to Protein Model Database in France during August' 2007 (Protein ID: PM 0074981).

\section{Discussion:}

Five theoretical models of DGAT Type 2A and Type 2B were generated by MODELLER. The generated models of DGAT Type 2A and Type 2B are represented in Figure 1(A) and $1(\mathrm{~B})$, respectively. Among the five DGAT Type 2B models, the lowest energy structure was DGAT2A 1 and DGAT2B as shown in Figure 1(A) and 1(B), respectively. In order to select the best model, we checked the structural validity of DGAT Type $2 \mathrm{~B}$ by PROCHECK. The torsion angles $\Phi$ and $\Psi$ of $88.9 \%$ of the residues had values within the most favored regions and only $0.3 \%$ of the residues had values within disallowed regions. The overall G-factor is a measure of the overall normality of the structure and low Gfactors indicate that residues have unlikely conformations. The overall value is obtained from an average of G-factors for all residues in structure. X-ray structure of DGAT Type $2 \mathrm{~A}$ with resolution of $2.0 \AA$ and a G-factor of $0.26 \AA$. The stereo-chemical quality of a protein model can be judged by the use of $\Phi$. and $\Psi$ scatter plots. The incorrect structures generally have a larger fraction of residues in the disallowed regions [18]. The model of DGAT Type $2 \mathrm{~B}$ has only $0.3 \%$ of its residues in the disallowed regions. Thus, the model satisfies criteria of a reasonably acceptable model.

The catalytic mechanisms of DGAT in various fungi are similar. However, they show significantly different substrate specificities in various species. The active site of DGAT Type 2B was hypothecated into two regions: (a) catalytic site and (b) primer binding site. The mechanism of action of DGAT involves a catalytic triad composed of an asparagine, a histidine, and the catalytic cysteine residues in the catalytic site. The catalytic triad of DGAT comprises of Cys112, His238, and Asn268. These residues are conserved in DGAT Type 2B (Cys113, His246, and Asn276). The enzyme DGAT Type 2B catalyzes carbon-carbon bond formation by condensing an acyl primer with an elongating carbon source often attached to a holo-ACP [19]. Hence, the primer binding site of DGAT is important to defining of substrate specificities. The isoformic structure was described elsewhere using the purification data reported by Kamisaka et al. [14]. It should be noted that the function of Type $2 \mathrm{~A}$ is still unclear and inter conversion of inactive to active form of DGAT probably requires certain hormonal and/or biochemical and/or molecular modulator. We docked Sphingosine, Trifluoroperazine, PhosphatidicAcid, Lysophospatidylserine, $\mathrm{KCl}$ and 1, 2-diolein to DGAT type 2B. These are lavonoids (a group of polyphenolic compounds) are widely distributed through out the plant kingdom with antibacterial activity. The interactions of these compounds with DGAT type $2 \mathrm{~B}$ are described.

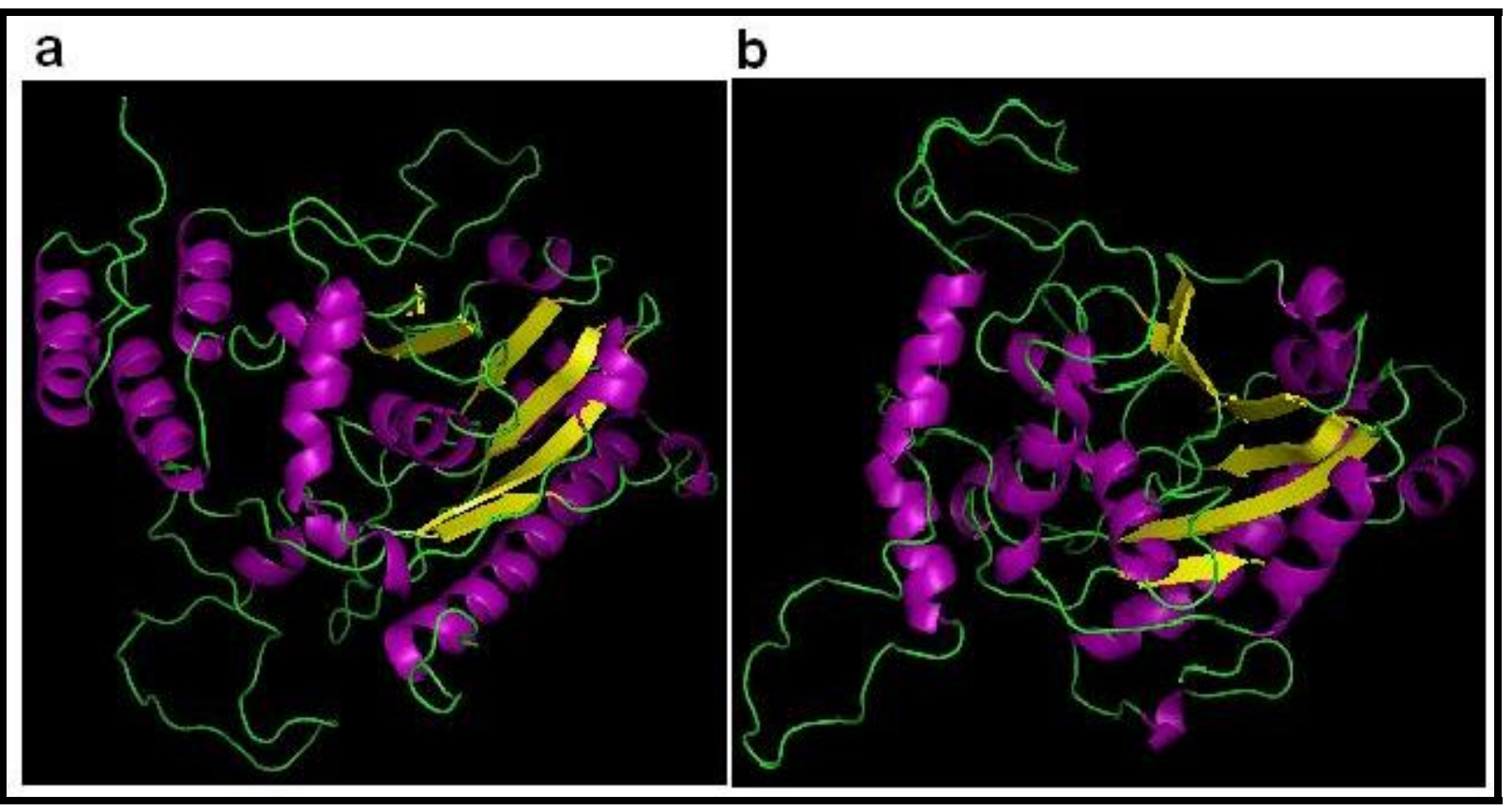

Figure 1: Model of Maretierella ramanniana DGAT (a) type 2A and (b) type 2B developed using MODELLER. 
a

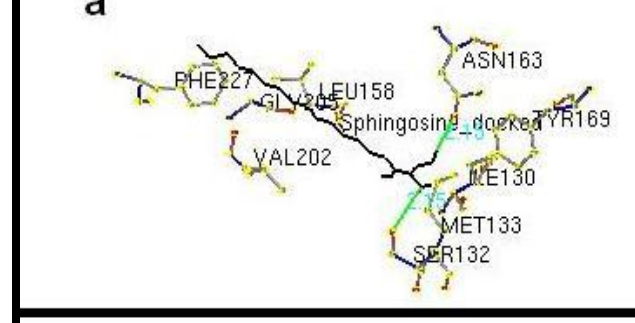

c

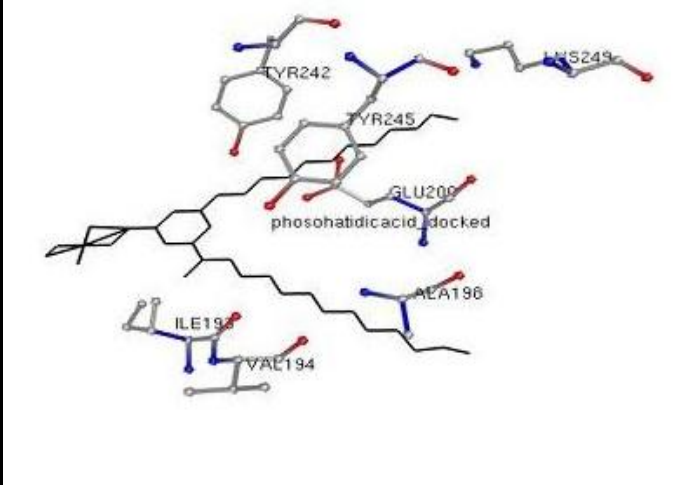

e

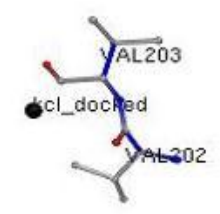

b

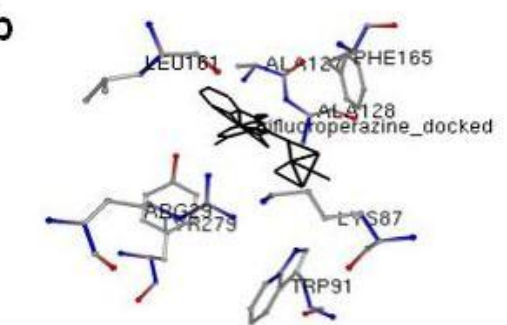

d
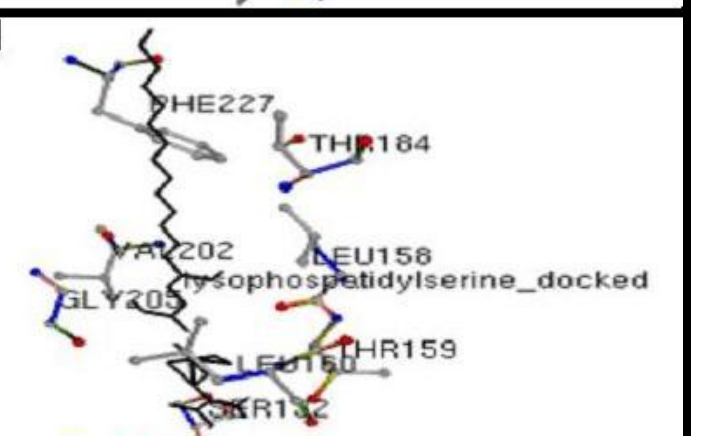

G\&Y129

JLE 130

\.

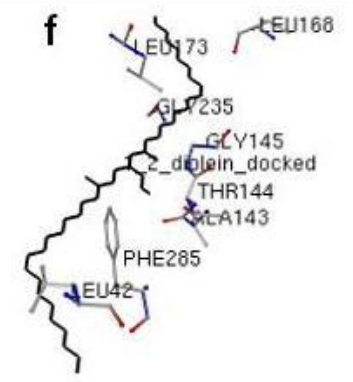

Figure 2: Docking models of several inhibitors with DGAT2B (a) Sphingosine; (b) Trifluoroperazine; (c) Phosphatidic acid; (d) Lyso phospatidylserine; (e) $\mathrm{KCl}$ and (f) 1, 2-diolein.

Ligand docking was carried out for DGAT Type 2B with these inhibitors (Figure: $2 \mathrm{~A}-2 \mathrm{~F}$ ). Lowest models of DGAT Type 2B in complex with inhibitors are shown in Figure 2A $2 \mathrm{~F}$. As shown in figure $2 \mathrm{~B}$, the side chains of Cys113, Phe308, Ser153 and Asn249 play important roles on hydrogen bonds with 7-, 4- and 4-hydroxyl groups of naringenin, respectively. However, in the case of phosphatidic acid, only the side chain of Ser153 forms hydrogen bond with 7-hydroxyl group of apigenin. The double bond of $\mathrm{C} 2-\mathrm{C} 3$ position of $\mathrm{C}$ ring in phosphatidic acid provides a structural rigidity compared with lysophospatidylserine. This rigidity interrupted an $\mathrm{H}$-bond between DGAT Type 2B and Lysophospatidylserine. The results described here is in close resemblance to the data described elsewhere [14]. It is speculated that the outer membrane of fungus acts as a barrier against lysophospatidylserine. However, it has already been reported that the purified DGAT utilizes a broad range of molecular ISSN 0973-2063 (online) 0973-8894 (print)

Bioinformation 3(9): 394-398 (2009) species of diacyl glycerol and acyl-CoA as substrates [14]. The highest activity of the purified enzyme was observed with sn-1, 2-diolein and lauroyl-CoA. Data show that anionic phospholipids such as phosphatidic acid (PA) activated the purified enzyme. Reports also show that sphingosine dose dependently inhibited DGAT activity activated by PA and basal activity without PA. These results explain for the molecular function of DGAT and the mechanism of triacylglycerol biosynthesis.

\section{Conclusion}

The three-dimensional structure model of DGAT Type 2B was created and described. Ligand docking shows that lysophospatidylserine provides the proper binding model in active site of DGAT Type 2 B with intensive hydrogen bonding network. Nevertheless, the comprehensive studies pertaining to elucidation of structural and functional models of Type 2A of DGAT as well as other TG biosynthesis 396 
enzymes may provide new insights into the molecular mechanism of these key players in fungal biotechnology.

\section{Acknowledgements:}

An institutional research promotion grant to the Department of Biotechnology, College of Engineering and Technology, Moradabad, U.P., India is acknowledged. The authors are grateful to Prof. R. M. Dubey (Managing Director, CET, IFTM, Moradabad, U.P, India) for providing the necessary facilities and encouragement. The authors are also thankful to Prof. B.N. Basu (Director, Academics) and Prof. B.N. Kaul (Director, Administration), College of Engineering and Technology, Moradabad, U.P., India, for their generous help and suggestions during the course of experimental work and manuscript preparation. The sincere moral support by Prof. A Srivastav (Head, Department of Mechanical Engineering), Caledonian College of Engineering, Muscat, Sultanate of Oman is duly acknowledged by the authors.

\section{References:}

[1] R.M. Bell \& R.A. Coleman, The Enzymes (Boyer, P.D., Ed.), 16: 111 (1993)

[2] L.B.M. Tijburg et al., Biochim. Biophys. Acta, 1004: 19 (1989) [PMID: 2663077]

[3] M. Frentzen, Lipid Metabolism in Plants, (Moore, T.S., Jr., ed.) CRC Press, Boca Raton, 195 (1993)

[4] C. Ratledge, Biotechnogy of oils and fats in Microbial lipids (Ratledge, C. and Wilkinson, S.G., eds.), Academic Press, London, 2:567 (1989)

[5] F. Manganaro \& A. Kuksis, Can. J. Biochem. Cell Biol., 63: 107 (1985) [PMID: 3995403]

[6] P. Kwanyuen and R.F. Wilson, Biochem. Biophys. Acta, 877: 238 (1996)
[7] M. Andersson et al., J. Lipid Res., 35: 535 (1994) [PMID: 8014588]

[8] O. Suzuki, Production of $\gamma$-linolenic acid by fungi and its industrialization in Biotechnology for Fat and oil industry (Applewhite, T.E., ed.), American Oil Chemists' Society, Champaign, 110:116 (1988)

[9] T. Yokochi and O. Suzuki, J. Jpn. Oil Chem. Soc. (Yukagaku), 38: 1007 (1989)

[10] T. Nakahara et al., Industrial Applications of Single Cell Oils (Kyle, D.J. and Ratledge, C., eds.) American Oil Chemists' Society, Champaign, 61:97 (1992)

[11] Y. Kamisaka et al., Lipids, 28: 583 (1993)

[12] Y. Kamisaka et al., J. Biochem., 116:1295 (1994) [PMID: 7706220]

[13] Y. Kamisaka And T. Nakahara, J. Biochem., 119: 520 (1996) [PMID: 8830048]

[14] Y. Kamisaka et al., J. Biochem. (Tokyo, 121:1107 (1997) [PMID: 9354384]

[15] S.F. Altschul et al., Nucleic Acids Res., 25: 3389 (1997) [PMID: 9254694]

[16] L.J. McGuffin and D.T. Jones, Bioinformatics, 19: 874 (2003) [PMID: 12724298]

[17] T.L. Sali and Blundell, J. Mol. Biol., 234:779 (1993). [PMID: 8254673]

[18] K.W. Jeong et al., Bull. Korean Chem. Soc., 28: 8: 1335 (2007)

[19] M.G. Pillai et al., Biochim. Biophys. Acta., 1393:128. (1998) [PMID: 9714775]

[20] N. Dwivedi et al., Bioinformation 2(8): 363 (2008) [PMID: 18685726]

[21] http://www.ncbi.nlm.nih.gov/entrez/

[22] http://mi.caspur.it/PMDB/

Edited by $P$. Kangueane

Citation: Mishra et al, Bioinformation 3(9): 394-398 (2009) License statement: This is an open-access article, which permits unrestricted use, distribution, and reproduction in any medium, for non-commercial purposes, provided the original author and source are credited. 


\section{Supplementary material}

\begin{tabular}{|c|c|c|c|c|c|c|}
\hline Inhibitors & $\begin{array}{l}\text { Energy } \\
\mathrm{Kcal} / \mathrm{mol}\end{array}$ & RMS & $\begin{array}{l}\text { Free } \\
\text { energy } \\
\text { Kcal/mol }\end{array}$ & $\mathbf{K I}$ & $\begin{array}{l}\text { Inter } \\
\text { molecular } \\
\text { energy }\end{array}$ & $\begin{array}{l}\text { Internal } \\
\text { energy }\end{array}$ \\
\hline Sphingosine & -17.45 & 54.06 & -13.15 & $2.31 \mathrm{e}-010$ & -18.13 & 0.68 \\
\hline Trifluoroperazine & -0.61 & 57.48 & -15.31 & $6.02 \mathrm{e}-012$ & -17.18 & 16.56 \\
\hline PhosphatidicAcid & -10.99 & 57.48 & -3.73 & 0.0 & -11.51 & 0.52 \\
\hline Lysophospatidylserine & -16.22 & 51.09 & -10.39 & $2.4 \mathrm{e}-008$ & -16.62 & 0.4 \\
\hline $\mathrm{KCl}$ & -1.22 & 54.43 & -1.22 & 0.13 & -1.22 & 0.0 \\
\hline
\end{tabular}

Table1: Docking data of the protein DGAT 2A with different inhibitors is given.

\begin{tabular}{lcc}
\hline \multicolumn{1}{c}{ Inhibitors } & \multicolumn{2}{c}{ Docked energy (kcal/mol) } \\
\cline { 2 - 3 } & DGAT type 2A & DGAT type 2B \\
\hline Sphingosine & -17.45 & -16.26 \\
Trifluoroperazine & -0.61 & -1.51 \\
Phosphatidic Acid & -10.99 & -12.59 \\
Lysophospatidylserine & -16.22 & -16.27 \\
KCl & -1.22 & -1.09 \\
1, 2-diolein & -16.02 & -14.58 \\
\hline
\end{tabular}

Table 2: Screening data generated using AUTODOCK v 3.0.5. Docking energies of different inhibitors with diacylglycerol acyltransferase (DGAT) type 2A and type 2B from Umbelopsis ramanniana is given. 